\title{
Effects of Gloves on the Grip Strength Applied to Cylindrical Handles
}

\author{
Bryan Wimer, Thomas W. McDowell, Xueyan S. Xu, \\ Daniel E. Welcome, Christopher Warren, and Ren G. Dong,
}

Engineering and Control Technology Branch, Health Effects Laboratory Division, National Institute for Occupational Safety \& Health, Morgantown, WV, USA

\section{INTRODUCTION}

Anti-vibration (AV) gloves have been used to reduce hand-transmitted vibration exposure. However, some workers have complained of increased hand and arm fatigue when they use these gloves. This is likely because some of the AV gloves could greatly reduce the grip strength or require workers to exert more grip effort in the operation of a vibrating tool. Although this has been generally understood, it is unknown exactly how much the AV gloves could reduce the grip strength applied to cylindrical handles used on many powered hand tools. It is also unclear whether the AV gloves could reduce grip strength more than regular work gloves. To answer these questions, this study measured the total grip strength applied on two cylindrical handles with, and without, wearing different gloves.

\section{METHOD}

The grip strength on a cylindrical handle was computed by quantifying the total contact force normal to the handle surface ${ }^{1}$. Two instrumented cylindrical handles (30 $\mathrm{mm}$ and $40 \mathrm{~mm}$ diam.) were used to measure the contact force. The $30 \mathrm{~mm}$ handle was comprised of an aluminum cylinder equipped with a Tekscan pressure mat ${ }^{1}$. The $40 \mathrm{~mm}$ handle was a six-piece handle developed in an earlier study ${ }^{2}$, which measured the contact force by means of shear strain gauges installed on each of the six arms. As shown in Fig. 1, four types of AV gloves and two types of standard work gloves were used in the experiment. Ten healthy male adult subjects participated in the experiment. The test setup and

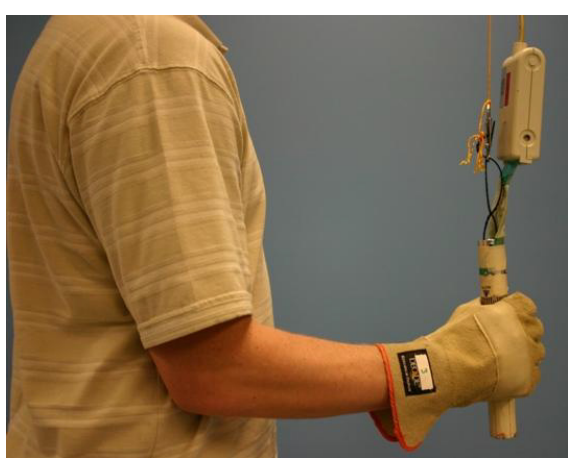

Fig.2. Test setup and subject posture used in the measurement.
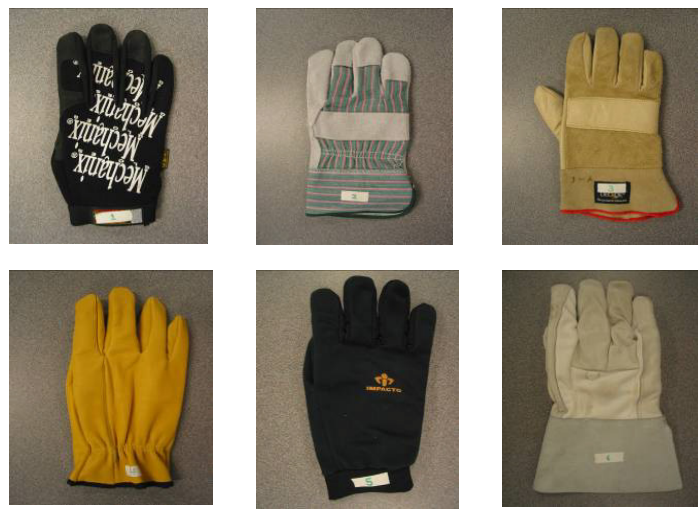

Fig. 1. The six gloves used in the study: (1) Mechanix Wear automotive (2) leather/cotton construction (3) Decade gel padded, antivibration (4) Impacto leather, air pocket, antivibration (5) Impacto mesh, air pocket, antivibration (6) ErgoAir pump, air pocket, antivibration subject posture are shown in Fig.2. Each subject was asked to apply his maximum grip for 5 seconds. The sampling rate was $5 \mathrm{~Hz}$. The middle 3 seconds of data were used to calculate the grip strength. Two test segments were carried out; one set of trials used the $40 \mathrm{~mm}$ handle and the other utilized the $30 \mathrm{~mm}$ handle. During each segment, the subject completed three maximum grip efforts on the handle with each of the six gloves as well as in the bare-handed condition for a total of 21 trials ( 7 hand conditions $\times 3$ trials). The test sequence in each segment was randomized among the subjects. The thickness of each glove was also measured. 


\section{RESULTS AND DISCUSSION}

As shown in Fig. 3, Glove 1 reduced the grip strength on both handles by less than $10 \%$ when compared with the bare hand grip strength. Each of the four anti-vibration gloves (Gloves 3-6) reduced the grip strength by more than $29 \%$, regardless of handle size. One of the standard work gloves (Glove 2$)$ also largely reduced the grip strength $(\geq 25 \%)$.

The major underlying cause of the observed strength reduction is likely associated with the mechanics of the glove in the gripping action. A glove is usually bowed and compressed at the palm side, and stretched at the back side. The forces required to generate these deformations must be the result of the hand grip effort. Therefore, part of the hand energy or grip effort must be absorbed by the glove. This mechanism suggests that the stiffness of a glove is likely to play an essential role in the reduction of the measured

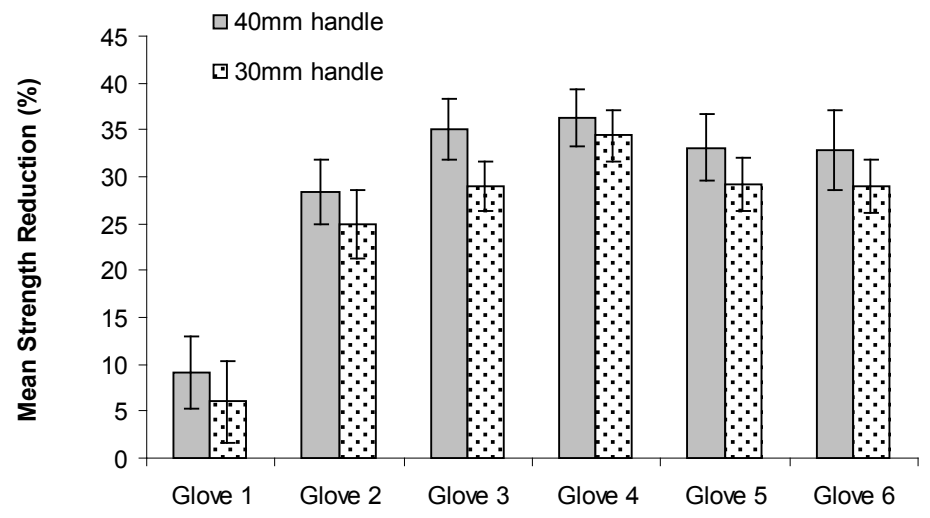

Figure 3. Mean grip strength percent reduction for the six gloves used in testing. (Y-error bars indicate the $95 \%$ confidence intervals for each mean). grip strength. The glove stiffness generally increases with the thickness in the glove material. This explains why the grip strength was reliably correlated with the measured glove thickness.

Glove use also increases the effective handle diameter, as confirmed from the glove influence on the grip force distribution around the circumference of the cylindrical handle. The changed handle size could also positively or negatively affect grip strength, depending on the original handle size. The increase of the handle size from $30 \mathrm{~mm}$ using gloves should theoretically increase the grip strength, but instead it was reduced. It is likely that the increased grip effort required to generate more deformation of the glove on a smaller handle counteracts the positive effect of the increased handle diameter.

These observations suggest that it is not the type of glove but the actual stiffness of the glove that primarily determines the reduction in grip strength. Because some AV gloves are thicker or stiffer than most standard working gloves, their reduction effect could be greater than that of the regular gloves. How to lessen the stiffness of AV gloves or to have less influence on the grip strength remains an interesting and useful research topic.

\section{REFERENCES}

1. Dong, R.G., Wu, J.Z., Welcome, D.E., and McDowell, T.W., 2008. A New Approach to Characterize Grip Force Applied to a Cylindrical Handle. Medical Engineering \& Physics, 30(1): 20-33.

2. Wimer, B.M., Dong, R.G., Welcome, D.E., Warren, C., and McDowell, T.W., 2009. Development of a New Dynamometer for Measuring Grip Strength Applied on a Cylindrical Handle. Medical Engineering \& Physics, 31(6): 695-704. 